\title{
The impact of pulse currents on morphological changes in lung and heart tissue under chronic purulent pneumonia
}

\begin{abstract}
We examined outbred rats with a model of chronic purulent pneumonia. We detected the damages to the interstitial tissue with the development of granulations and foci of inflammation, pneumosclerosis in the lung tissue. Uneven hypertrophy of cardiomyocytes, thickening of the walls of blood vessels and an increase in their quantitative ratio, moderate proliferation of connective tissue layers in the perivascular zones, intermuscular edema with impaired circulation of the muscle wall were manifested in the heart tissue. After electrical myostimulation pulse currents of the respiratory muscles in the lung tissue revealed increased amount of connective tissue in the septa, increase in the number of macrophages, and in cardiac tissue focal dissociation of cardiomyocytes, irregular interstitial edema, hypertrophy of the walls of arterioles, hyperemia, poor circulation in the appearance of focal hemorrhages in the myocardium and epicardium.
\end{abstract}

Keywords: chronic purulent, tissue, lung inflammation, heart, morphology, experiment, laboratory animals, pulse currents, electromyostimulation
Volume 5 Issue 2 - 2020

\section{Sadykova GA,' Rakhmatullaev HU,' Zalyalova ZS, ${ }^{2}$ Akhmedov MA' \\ 'Republican specialized scientific and practical medical center of therapy and medical rehabilitation, Ministry of health of the Republic of Uzbekistan Tashkent, Uzbekistan \\ ${ }^{2}$ Tashkent Institute of advanced medical training, Uzbekistan}

Correspondence: Sadykova GA,Republican specialized scientific and practical medical center of therapy and medical rehabilitation,Ministry of health of the Republic of Uzbekistan, Uzbekistan, Tel +998909857665, Email gulora@yandex.ru

Received: February 17, 2020 | Published: April 06, 2020

\section{Introduction}

Chronic obstructive pulmonary disease (COPD) remains an extremely common disease, with mortality continuing to increase. ${ }^{1-5}$ Skeletal and respiratory muscle dysfunction is one of the most common systemic effects in patients with chronic obstructive pulmonary disease (COPD). The use of neuromuscular electrical stimulation is an important rehabilitation program for patients with COPD with severe physical disabilities. Skeletal muscle dysfunction associated with functional and atrophic changes leads to a decrease in strength and endurance, and changes in the activity of enzyme systems. Due to pneumonia, the cardiovascular system stops functioning properly, worsening the prognosis of recovery. One of the rare causes of shortness of breath, which is not explained by other diseases, is a dysfunction of the diaphragm. A significant role in the development of respiratory failure in COPD patients is played by increasing weakness of the respiratory muscles, mainly the diaphragm. ${ }^{6}$ Issues of primary and secondary prevention, as well as medical rehabilitation of COPD require further development with the use of modern new technologies and non-drug treatment methods. ${ }^{7}$

\section{Goal}

Study of the effect of pulse currents on morphological changes in lung and heart tissue in an experiment based on a model of chronic purulent inflammation of the lungs.

\section{Material and methods}

Outbred white male rats were examined. The model was reproduced according to the method of Batyrova $\mathrm{ZB}$ et al. ${ }^{8}$ which is based on prolonged mechanical intra-tracheo-bronchial irritation of the respiratory tract. Under sterile conditions, under local novocaine anesthesia, a longitudinal incision was made along the midline on the anterior surface of the neck of the animal $1.5-2.0 \mathrm{~cm}$ long. After exposure of the anterior wall of the trachea between its rings, a capron thread with a diameter of $0.4 \mathrm{~mm}$, up to $8-10 \mathrm{~cm}$. In this case, the distal end of the thread was in the lumen of the trachea, and its proximal end was fixed on the skin. The wound was sutured in layers tightly. After 30-45 days from the start of the experiment, the fixing kapron thread was cut and the thread was removed with tweezers. Slaughter of animals was carried out by instant decapitation on 30-45 days from the moment of reproduction.

For morphological studies taken after slaughter, pieces of the heart and lung tissue were fixed in a $10 \%$ solution of neutral formalin. Paraffin histological sections 5-6 $\mu \mathrm{m}$ thick were stained with hematoxylin eosin. Microscopy of the preparation was carried out under an optical microscope XS-213 and a microscope from Leica.

All subjects were weighed animals, temperature measurement, heart rate calculation, BPD, General blood test for biochemical studies. After 30-45 days from the beginning of the experiment, by cutting the outer wall of the skin above the trachea, the fixing nylon thread was removed and the latter was extracted with tweezers without opening the trachea. Experimental animals are divided into three groups according to the treatment method:

No. 1. Pulse current treatment was performed from the Stimul-1 device in the rectified mode. Electrodes of $1,5 \times 1,3 \mathrm{~cm}$ in size were attached to the pre-treated skin surface of e-CPI along the middle axillary line bilaterally at the level of 7-8 ribs. the current Strength is $0.1 \mathrm{~mA} / \mathrm{cm} 2$, the frequency is $2.5-5.0 \mathrm{Ms}$.

No. 2. Treatment with pulse current in alternating mode from the device "Stimulus-1". The current strength of $0.1 \mathrm{~mA} / \mathrm{cm} 2$, a frequency of 2.5 to $5.0 \mathrm{Ms}$. For 5 minutes, e/d, number 8 procedures.

No. 3. Pulse current treatment was obtained from the developed program "eShifo" embedded in the "Smartphone". The current was supplied with a frequency of F-50 Hz, a modulation depth of 
$\mathrm{W}-100$ and a power of V-10v.5 min, e/d, no. 8. The control group consisted of 6 rats with e-CPI (they did not receive treatment). Animal slaughtering was performed by instant decapitation for 30-45 days from the moment of reproduction of the model-e-HVL. For 5 minutes, e/d, number 7 procedures.

\section{Research results}

Microscopic examination of the organs of experimental animals revealed that animals with clinically pronounced pathology of the respiratory system, in particular the presence of shortness of breath and wheezing, in the lungs were determined changes characteristic of focal pneumonia, namely: thickening of the walls of the bronchioles due to edema and round-cell infiltration with a predominance of lymphocytes, hyperplasia of peribronchial lymphoid tissue, focal desquamation of the epithelium of the bronchioles in the lumen, full blood vessels. In the lumen of some bronchioles, mucous plugs were identified, homogeneously eosinophilic colored, which further aggravated the processes of violation of pulmonary ventilation. The walls of the bronchioles were spasmed with bizarre scalloped edges, sclerosis of the walls and hyperplasia of the epithelial cells lining the bronchus. The walls around the bronchioles were infiltrated by lymphoid-histiocytic-leucocytic elements. Due to the presence of a pathological inflammatory process in the lung tissue were determined (Figure 1).

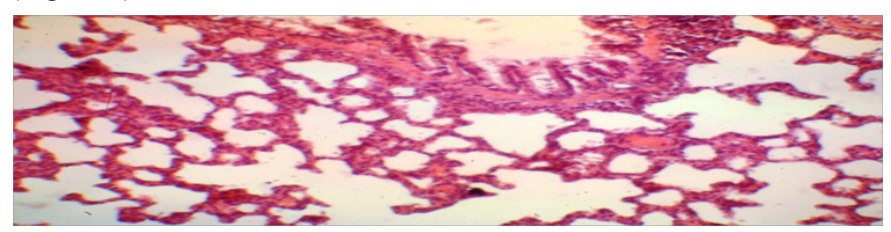

Figure I The rat's lung is normal. The wall of the lobular bronchus with a secretory cylindrical epithelium. Color: hematoxylin-eosin. Lead away. 10*10, $10 * 20$.

In animals with clinically pronounced respiratory pathology, in particular the presence of shortness of breath and wheezing, microscopic examination of the organs of experimental animals on the day of the experiment, in contrast to a group of healthy animals, revealed changes in the lungs characteristic of focal pneumonia. The lumen of the bronchi was filled with a transparent viscous liquid, there was swelling of the lung, the lungs were hyperemic with foci of suppuration against the background of the tuberosity of the lung. On the incision, a purulent thick greenish liquid was released in the areas of suppuration. Microscopically, thickening of the walls of the bronchioles due to edema and round-cell infiltration with a predominance of lymphocytes, hyperplasia of peribronchial lymphoid tissue, focal desquamation of the epithelium of the bronchioles into the lumen, and full blood vessels were detected. In the lumen of some bronchioles, mucous plugs were identified, homogeneously eosinophilic colored, which further aggravated the processes of violation of pulmonary ventilation. There were no visible changes on the part of other internal organs. Arterial and capillary fullness of the septum vessels is clearly expressed (Figure 2).

Data of morphological changes in long-term bronchial irritation and the use of the e-HVL model indicate the tendency of the infection to penetrate into the depth and affect not only the bronchial mucosa, but also the muscle wall and peribronchial tissue (meso-and peribronchitis). Changes are mainly small bronchi; their walls are swollen, infiltrated, capillaries are stretched and filled with blood.
With the development of chronic pneumonia, various forms of changes occur in the lung tissue: pneumonic foci, sclerosis, focal emphysema, atelectasis, and bronchiectasia. This is due to the simultaneous involvement of the entire lung, bronchi, interstitial tissue, alveoli, and blood vessels in the process. But the leading process in the lungs is the lesion of interstitial tissue with the development of granulations and foci of pneumosclerosis. In the lungs of rats treated pulse stimulation of diaphragmatic muscle are determined by changes in the form of increased amount of connective tissue in the septa, increase in the number of macrophages, thickening of the walls of arterioles due to hypertrophy of the muscle membrane and increasing the amount of connective tissue.

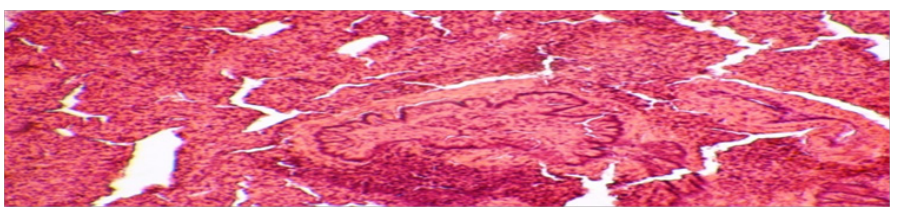

Figure 2 Rat lung with a model of chronic lung inflammation. Destructive and inflammatory changes in lung tissue. There are Muco-purulent plugs in the lumen of the bronchioles. Color: hematoxylin-eosin. Lead away. 10*10, $10 * 20$.

The morphological picture of the heart in rats in the control group of animals is characterized by unexpressed tissue hyperemia (Figure $3)$.

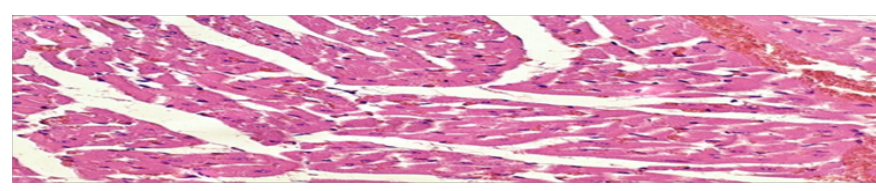

Figure 3 Heart of a control group rat. Hyperemia of the tissue. Lead away. $10 \times 10,10 \times 20,10 \times 40$. Hematoxylin-eosin staining.

In the heart, compared with the control group of animals, there was an uneven hypertrophy of cardiomyocytes, thickening of the vessel walls and an increase in their quantitative ratio (Figure 4).

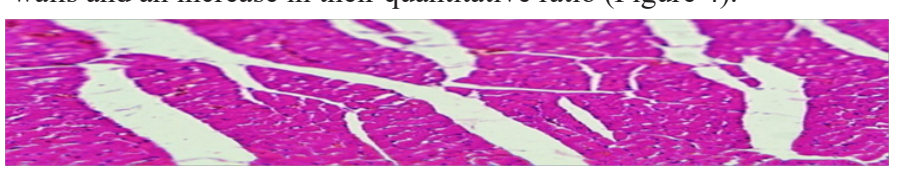

Figure 4 Heart, experienced animal I. Layers of tender connective tissue in the perivascular and intermuscular sections, intermuscular edema. Lead away. $10 \times 10,10 \times 20$. Coloring hematoxilin-eosin.

Moderate growth of connective tissue layers in perivascular zones and intermuscular edema was also determined. A hyperemia of a tissue, focal perivascular erythrocephalus and hypertrophy of the walls of arterioles.

Thus, the main group of rats №1,2 preserved focal pronounced inflammatory polymorphocellular infiltration and edema of the septum with focal atelectases, venous fullness, determined focal erythrodyapedesis in the tissue, focal emphysema, focal distelectases, focal desquamation of the epithelium of the bronchioles. The growth of peribronchial lymphoid tissue is insignificant. There is also a cluster of dense organizing masses in the lumen of some alveoli. In the same group, another similar rat has unexpressed hyperplasia of peribronchial lymphoid tissue, thickening of the walls of arterioles, focal emphysema, but there is a sharp decrease in inflammatory infiltration, but there are focal hemorrhages. 
In the main group of rats treated in the "eShifo" group, full blood is determined, focal extensive hemorrhages in the septum and lumen of the alveoli. Thus, a rat in this group had mild inflammatory infiltration against the background of severe lymphoid tissue hyperplasia, but there were hemorrhages and focal emphysema. Moderate roundcell infiltration of diffuse septa is preserved, mucous plugs in some bronchioles are detected, and there is practically no hyperplasia of peribronchial lymphoid tissue. In the control group of untreated rats, diffuse infiltration of the interalveolar septa, no lymphoid tissue at all, pronounced hypertrophy, distelectases, focal emphysema, septum edema, uneven spasm of the walls of the bronchioles.

After electromyostimulation of respiratory muscles in e-CPI treatment with pulse currents in experimental groups, dystrophic swelling and focal dilatation of cardiomyocytes, uneven inter-daily edema, hypertrophy of arteriole walls, full blood, sometimes paretic expansion of capillaries with stasis of red blood cells in the lumen are determined. Also, manifestations of circulatory disorders are expressed in the form of focal hemorrhages in the myocardium and epicardium.

\section{Connection with literary sources}

Among the systemic complications of chronic obstructive pulmonary disease, this insidious pathology, is skeletal muscle dysfunction. Dysfunction progresses in parallel with a decrease in the functional reserve of the lungs and an increase in shortness of breath, leading to even greater detrenirovannosti patients and reduced tolerance to physical activity. For a long time, the importance of muscle dysfunction in COPD was underestimated by doctors due to insufficient research on this issue. However, today there are concrete data that allow us to rethink the significance of muscle dysfunction in the clinic of chronic obstructive pulmonary disease According to research by a number of authors, electrical stimulation of the diaphragm and diaphragmatic muscles significantly improves the function of external respiration in patients with bronchial asthma and chronic obstructive bronchitis.

Respiratory muscle weakness is one of the most important causes of community-acquired pneumonia in elderly people. Vacuole dystrophy of myocytes, myocytolysis, and hemorrhages were found in those who Died from severe influenza pneumonia. In some patients of this group, rhabdomyolysis was the leading mechanism of respiratory muscle damage.

In the literature review, an attempt is made to summarize the data of fundamental and clinical studies on the problem of respiratory muscle dysfunction to determine the pathophysiological patterns and clinical features of its various variants in the most common lung diseases. Further research in this area should ensure the development of modern means of pharmacological correction of respiratory muscle dysfunction. In connection with the above information, our research is aimed at finding out the possibility of correcting respiratory muscle fatigue in pulmonary pathology and is a pilot study. Research continues.

\section{Conclusion}

Thus, long-term mechanical irritation of the bronchi causes, along with chronic purulent inflammation of the lungs, morphological changes also in the heart tissue, indicating the inclusion in the inflammatory process in e-CPI along with the lung tissue and heart tissue with cellular shifts in them. After stimulation with pulsed currents in alternating and constant modes, hyperplasia of lymphoid tissue increases, which causes a decrease in inflammatory phenomena in the lung tissue. In rats treated with a nested program via a smartphone, inflammatory manifestations also decrease in case of hyperplasia of lymphoid tissue, but the number of hemorrhages in the lung tissue increases. Pulse currents in this dosage cause circulatory disorders in the form of focal hemorrhages in the myocardium and epicardium.

\section{Ethical approval}

The research was carried out on an applied grant approved by the Ministry of health of the Ministry of health.

\section{Control group}

In the article published earlier, there is a control group without the influence of pulse currents.

\section{Acknowledgments}

None.

\section{Conflicts of interest}

None.

\section{References}

1. Global strategy for the diagnosis, treatment and prevention of chronic obstructive pulmonary disease (revised 2011) translated from English, edited by AS Belevsky, M-russian republican society-2012.- 80C, Il. COPD: Morphological picture. Edited by AG. Chuchalin. 2008;21:35.

2. Landysheva IV. Chronic pulmonary heart. IV Landysheva, S Yu Landyshev. Blagoveshchensk. 2001:140.

3. Sadykova GA, Aripov BS. The state of the tonus of the respiratory muscles in patients with chronic obstructive pulmonary disease. Proceedings of the international scientific and practical conference. Dushanbe. 2019(2):177-179.

4. Debigaru R. Ubiquitination and proteolysis in limb and respiratory muscles of patients with chronic obstructive pulmonary disease. Proc Am Thorac Soc. 2010;7(1):84-90.

5. Sadykova GA, Rakhmatullaev Zalyalova Z, et al. Morphological changes of the diaphragm in chronic purulent pneumonia (experimental study). Acta Scientific Medical Sciences Jurnal. 2019;3(3):60-63.

6. Sadykova GA, Rakhmatullaev HU, Zalyalova ZS. Morphological changes of the diaphragm in chronic purulent inflammation of the lungs (experimental study). Therapeutic Bulletin of Uzbekistan. 2018;4:53-57.

7. Shmelev EI. Chronic obstructive pulmonary diseases and chronic pneumonia. Terminological and clinical aspects. Rus honey journal. 2000;12:487-491.

8. Batyrova ZB, Shamirzaev NH. Method for modeling chronic non-specific lung inflammation (Certificate v 20420502 a 2). 